\title{
Chromatin decouples promoter threshold from dynamic range
}

\author{
Felix H. Lam ${ }^{1,2}$, David J. Steger ${ }^{1} \dagger \&$ Erin K. O'Shea ${ }^{1}$
}

Chromatin influences gene expression by restricting access of DNA binding proteins to their cognate sites in the genome $e^{1-3}$. Large-scale characterization of nucleosome positioning in Saccharomyces cerevisiae has revealed a stereotyped promoter organization in which a nucleosome-free region (NFR) is present within several hundred base pairs upstream of the translation start site $e^{4,5}$. Many transcription factors bind within NFRs and nucleate chromatin remodelling events which then expose other cis-regulatory elements $^{6-9}$. However, it is not clear how transcription-factor binding and chromatin influence quantitative attributes of gene expression. Here we show that nucleosomes function largely to decouple the threshold of induction from dynamic range. With a series of variants of one promoter, we establish that the affinity of exposed binding sites is a primary determinant of the level of physiological stimulus necessary for substantial gene activation, and sites located within nucleosomal regions serve to scale expression once chromatin is remodelled. Furthermore, we find that the $S$. cerevisiae phosphate response $(\mathrm{PHO})$ pathway exploits these promoter designs to tailor gene expression to different environmental phosphate levels. Our results suggest that the interplay of chromatin and binding-site affinity provides a mechanism for finetuning responses to the same cellular state. Moreover, these findings may be a starting point for more detailed models of eukaryotic transcriptional control.

When cells sense changes in environmental inorganic phosphate $\left(\mathrm{P}_{\mathrm{i}}\right)$, the activity of the transcription factor Pho4 is modulated by phosphorylation ${ }^{10}$ : Pho4 is phosphorylated on four sites, cytoplasmic and inactive when cells are grown in $\mathrm{P}_{\mathrm{i}}$-rich medium; it is phosphorylated selectively on one site and localized to the nucleus in intermediate $\mathrm{P}_{\mathrm{i}}$ (about $100 \mu \mathrm{M}$ ) conditions; and it is unphosphorylated, nuclear and fully active in $\mathrm{P}_{\mathrm{i}}$ starvation $^{11,12}$. The co-activator Pho2 interacts with unphosphorylated $\mathrm{Pho} 4$ and is required for induction of many $\mathrm{PHO}$ genes ${ }^{13,14}$; however, it is not thought to be regulated in response to $\mathrm{P}_{\mathrm{i}}$ availability ${ }^{12}$. Despite being controlled by the same activators, the target gene PHO5 is expressed at a low level in intermediate $\mathrm{P}_{\mathrm{i}}$ conditions, whereas $\mathrm{PHO} 4$ is significantly induced ${ }^{14}$. Although both promoters contain a combination of highand low-affinity Pho4-binding sites ${ }^{15-17}$, more Pho4 is recruited to PHO84 in intermediate $\mathrm{P}_{\mathrm{i}}$ conditions than to $\mathrm{PHO5}^{14}$. We hypothesized that chromatin may influence gene expression by differentially regulating the accessibility of Pho4 sites in the PHO5 and PHO84 promoters.

To test this hypothesis, we constructed variants of the PHO5 promoter controlling transcription of a green fluorescent protein (GFP) reporter gene (Fig. 1a). The PHO5 promoter contains five positioned nucleosomes (numbered -5 to -1 ), a low-affinity Pho4 site (CACGTTt) and a Pho2 site in the NFR, and a high-affinity
Pho4 site (CACGTGg) and distal Pho2 sites occluded under nucleosome -2 (refs 16-20). The difference in affinity between these two Pho4 sites is estimated to be about 3 -fold by electrophoretic mobility shift assay ${ }^{21}$, and about 13 -fold by a recent high-throughput fluorescence-based assay ${ }^{22}$. The Pho4 site in the NFR is required for transcriptional induction: Pho4 binds this site and recruits ATPdependent chromatin remodelling complexes that displace nucleosomes to expose additional cis-regulatory elements ${ }^{23,24}$. We manipulated the accessibility of Pho4 sites by relocating motifs into and/or out of nucleosomal regions and verified that our genetic perturbations have minimal impact on PHO5 chromatin structure (Supplementary Figs 1 and 2$)^{5}$.

To assess the effects of these alterations quantitatively, we assayed the steady-state expression of PHO5 promoter variants grown in different concentrations of $\mathrm{P}_{\mathrm{i}}$. We observed surprisingly stereotyped behaviour when the output profile for each variant was normalized to its maximum expression. When grown in intermediate $(10-100 \mu \mathrm{M})$ $\mathrm{P}_{\mathrm{i}}$ concentrations, variants containing a low-affinity motif in the NFR induce slightly ( $5 \pm 4 \%$ of maximum levels), whereas variants with an exposed high-affinity motif induce significantly $(37 \pm 2 \%$ of maximum levels) (Fig. 1b). This response is a function of the accessible Pho4 site and is independent of the number or placement of occluded Pho4 motifs: similar behaviour is observed when the highaffinity Pho4 site normally occluded by nucleosome -2 is transplanted or added under nucleosome -3 (variants L2, L3), or when there is a single accessible $\mathrm{Pho} 4$ site (variants L1, H1).

On the basis of our steady-state measurements, we expect promoter variants with an exposed high-affinity site to induce when cells reach the intermediate $P_{i}$ range, whereas we expect promoters with an exposed low-affinity site to remain essentially repressed until near the zero $\mathrm{P}_{\mathrm{i}}$ state. Indeed, we observe a difference in induction kinetics when the promoters are scaled to their particular dynamic range (Fig. 1c): the time-to-half-maximal induction of the high-affinity class is $146-157 \mathrm{~min}$, whereas that of the low-affinity class is 186197 min (Supplementary Table 1, interpolated values). The average $40 \mathrm{~min}$ lag observed in the low-affinity variants is not solely a result of inherently slower promoter activation; the kinetic difference is reduced by disabling the vacuolar phosphate buffer which accelerates the transition to the zero $\mathrm{P}_{\mathrm{i}}$ state (Supplementary Fig. 3 and Supplementary Table 1$)^{25}$. Rather, the lag is a consequence of how the affinity of the exposed site mediates a different response to the same upstream Pho4 signal in intermediate $\mathrm{P}_{\mathrm{i}}$ conditions. Thus, the affinity of the transcription-factor binding site(s) in the NFR also influences the apparent kinetics of gene expression.

To assess the generality of this promoter design principle, we asked if binding-site coordinates and affinities, in combination with nucleosome positions, would allow quantitative prediction of promoter

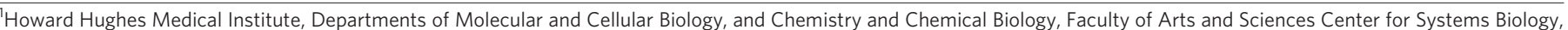

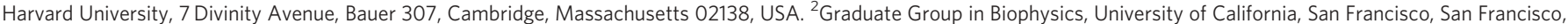
California 94158, USA. †Present address: Division of Endocrinology, Diabetes and Metabolism, 611 Clinical Research Building, University of Pennsylvania, 415 Curie Boulevard, Philadelphia, Pennsylvania 19104, USA. 
a
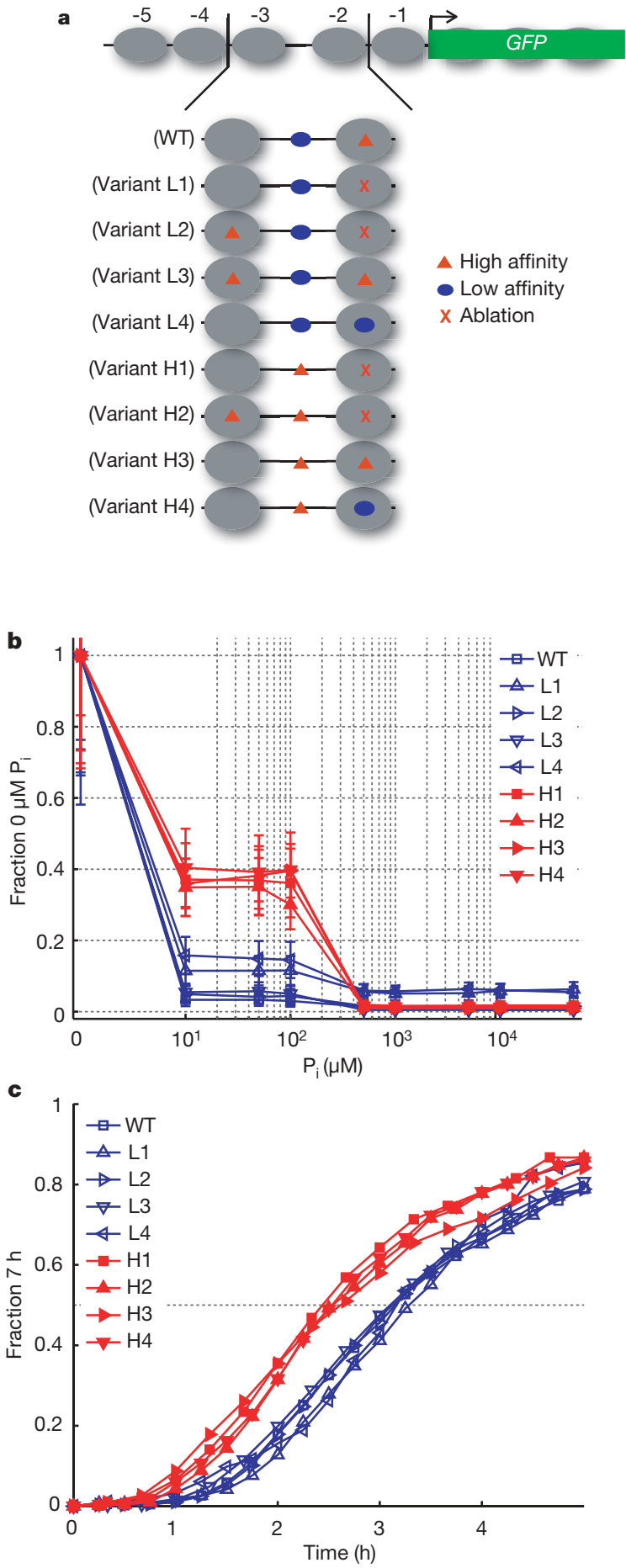

Figure 1 | PHO5 promoter variants and quantitative expression behaviour. a, Schematic of all $\mathrm{PHO} 5$ promoter variants controlling expression of yeGFP1. Large grey ovals represent nucleosomes, red triangles the highaffinity Pho4 motif (CACGTGg), blue ovals the low-affinity Pho4 motif (CACGTTt), and $\mathrm{X}$ the motif ablations. $\mathbf{b}$, Physiological transcriptional response to extracellular inorganic phosphate $\left(\mathrm{P}_{\mathrm{i}}\right)$ measured by flow cytometry. Data points represent median steady-state expression levels normalized to the median observed in $P_{i}$ starvation. Error bars represent interquartile ranges, which were observed to encompass the medians of at least three independent measurements. c, Induction kinetics in $\mathrm{P}_{\mathrm{i}}$ starvation. Data points represent median fluorescence levels scaled between the promoter-specific expression minimum at $0 \mathrm{~h}$ and maximum at $7 \mathrm{~h}$. For b and c, red traces designate variants with an exposed high-affinity site, and blue traces variants with an exposed low-affinity site. response profiles and kinetics. As a test set, we selected and mapped promoter chromatin (Supplementary Fig. 1) for PHO84 and five additional Pho4 targets that are highly induced by $\mathrm{P}_{\mathrm{i}}$ starvation and have a combination of high- and low-affinity motifs for Pho4 (Supplementary Figs 4 and 5). PHO89 and PHM6 exhibit nucleosomal arrays that, like PHO5, expose only a low-affinity Pho4 motif(s) in the repressed state (Fig. 2a). The remaining four genes (PHO84, PHO8, PHM2 and PHM4) have promoters containing at least one accessible high-affinity motif (Fig. 2b). Although many of these promoters likely contain Pho2 sites (which are difficult to recognize by sequence owing to AT-rich composition and degeneracy), in intermediate and high $\mathrm{P}_{\mathrm{i}}$ conditions these sites may not have a large influence on Pho4 recruitment because Pho4 is phosphorylated and unable to interact efficiently with Pho2 (ref. 12).

We expected PHO89 and PHM6 to behave similarly to PHO5, exhibiting low-level expression in intermediate $\mathrm{P}_{\mathrm{i}}$ and slow induction in $\mathrm{P}_{\mathrm{i}}$ starvation, and promoters containing at least one exposed highaffinity site ( $\mathrm{PHO} 84, \mathrm{PHO}$, $\mathrm{PHM} 2$ and $\mathrm{PHM} 4$ ) to exhibit substantial expression and rapid induction. We created GFP transcriptional reporter strains and, consistent with our expectations, observed that PHO89 and PHM6 induce minimally in intermediate $\mathrm{P}_{\mathrm{i}}$ conditions ( $3 \pm 1 \%$ of maximum) whereas PHO84, PHO8, PHM2 and PHM4 induce significantly ( $34 \pm 5 \%$ of maximum) (Fig. 2c). Additionally, when starved for $\mathrm{P}_{\mathrm{i}}$, we observed that genes with an exposed highaffinity motif reach half-maximal induction in 121-156 min compared with 198-222 min for the low-affinity class (Fig. 2d and Supplementary Table 2, interpolated values). Therefore, the affinity of the accessible Pho4 site is sufficient to define a promoter threshold from which steady-state and kinetic behaviour follow.

Nucleosome mapping and sequence analysis reveal that about $50 \%$ of the evolutionarily conserved $\mathrm{Pho} 4$ sites in our selection of $\mathrm{PHO}$ promoters are inaccessible in the repressed state and become exposed when chromatin is remodelled in response to $\mathrm{P}_{\mathrm{i}}$ starvation (Fig. 2a, b and Supplementary Fig. 5). To assess the influence of these sites on quantitative gene expression in a controlled sequence background, we analysed the maximum induction levels of the PHO5 promoter variants (Fig. 3 and Supplementary Table 3). Consistent with the role of the accessible site in the nucleation of remodelling, variants lacking exposed motifs (A1-A4) are uninducible or severely crippled. Otherwise, maximum transcriptional output differs by a factor of about four to seven and correlates with the number, affinity and placement of Pho4 sites, irrespective of their accessibility in the repressed state. Thus, chromatin enables significant decoupling of the determinants of promoter threshold from determinants of expression capacity.

Collectively, our observations are consistent with a model whereby nucleosomes compete with Pho4 for binding DNA, and nucleosome occupancy is dynamic and determined by a balance of assembly and disassembly activities ${ }^{26-28}$. The chromatin maps and range of promoter outputs observed in different $\mathrm{P}_{\mathrm{i}}$ conditions (Figs $1 \mathrm{~b}, 2 \mathrm{a}-\mathrm{c}, 3$, Supplementary Fig. 6 and Supplementary Table 3) likely reflect the different extents of chromatin remodelling resulting from a competition between Pho4 binding and nucleosome re-assembly. Even the A4 PHO5 promoter variant containing two high-affinity sites occluded by nucleosomes can be induced to a reasonable level (Fig. 3). This observation is consistent with the model that chromatin is dynamic, and that multiple buried high-affinity sites are sufficient for Pho4 to compete successfully with nucleosomes.

At the sub-maximal Pho4 activity (that is, nuclear concentration and/or phosphorylation state ${ }^{14,29}$ ) associated with intermediate $\mathrm{P}_{\mathrm{i}}$ conditions, the occupancy of Pho4 at an exposed high-affinity site may be sufficient to nucleate substantial chromatin remodelling such that regulatory sequences that were previously nucleosomal now become more accessible, allowing for appreciable transcription. However, promoters with an exposed low-affinity motif likely do not achieve similar Pho4 occupancy until $\mathrm{P}_{\mathrm{i}}$ starvation. Indeed, in vivo $\mathrm{Pho} 4$ occupancy is substantially higher in intermediate $\mathrm{P}_{\mathrm{i}}$ 
a
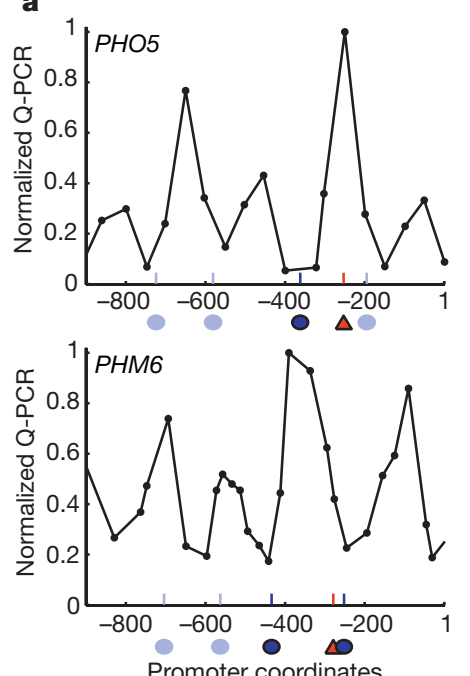

b
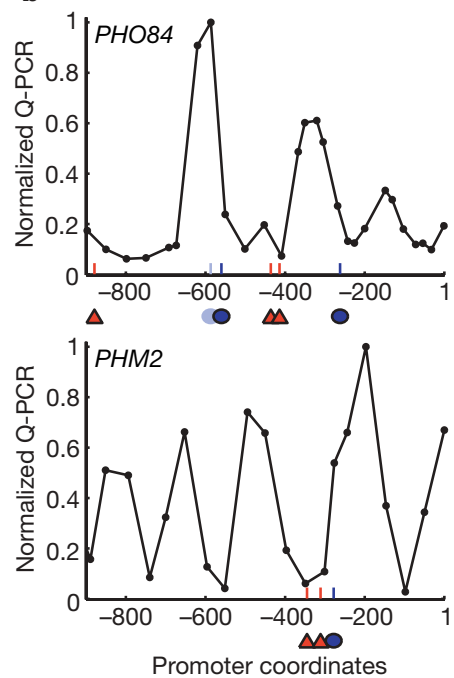



Promoter coordinates

c

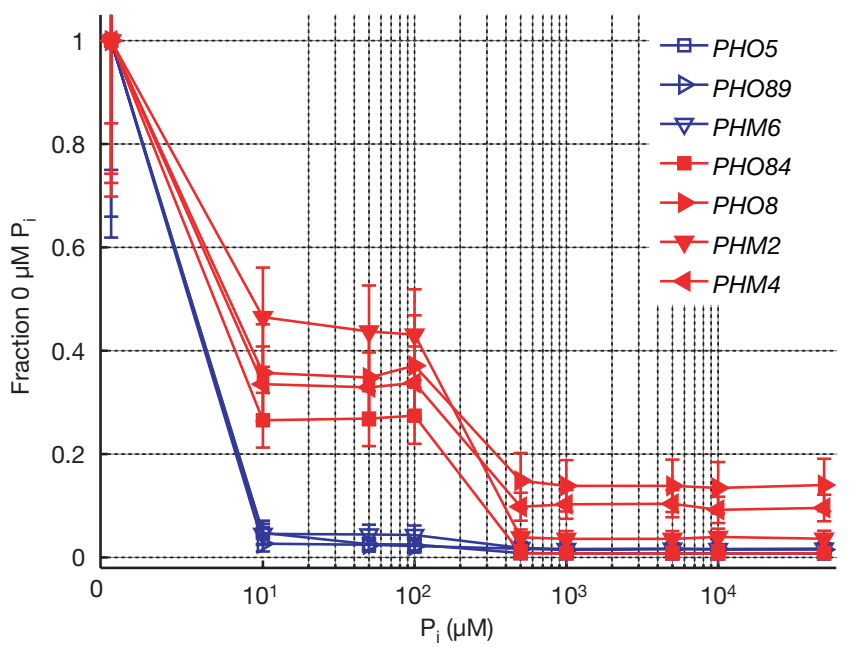

d
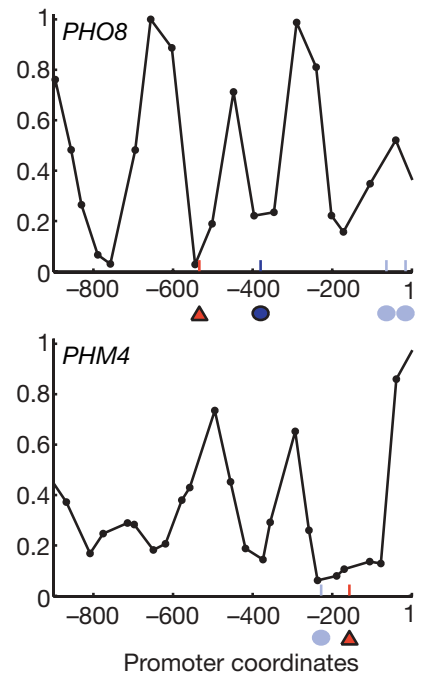

Figure 2 | Promoter architecture and quantitative expression behaviour of representative $\mathbf{P H O}$ genes. a, b, Promoter architecture schematized by superimposing nucleosome positions measured in repressing $\left(10 \mathrm{mM} \mathrm{P} \mathrm{P}_{\mathrm{i}}\right)$ conditions onto Pho4-binding sites identified through bioinformatic analysis (Supplementary Fig. 4). Red triangles represent evolutionarily conserved high-affinity motifs (CACGTG consensus), dark-blue ovals represent evolutionarily conserved low-affinity motifs (deviations from the

conditions at $\mathrm{PHO}$ promoters containing accessible high-affinity sites than at promoters with exposed low-affinity sites (Fig. 4a). Tuning Pho4 activity such that only promoters with exposed
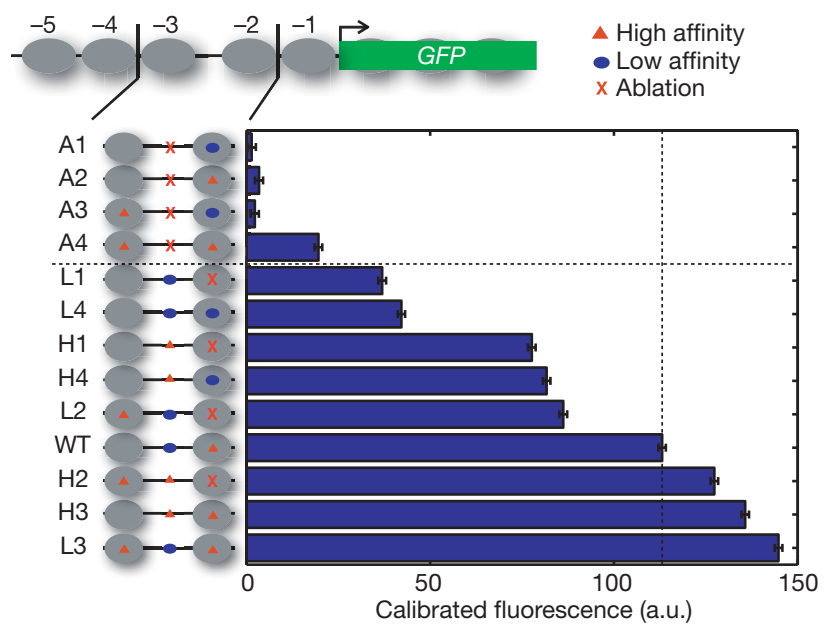

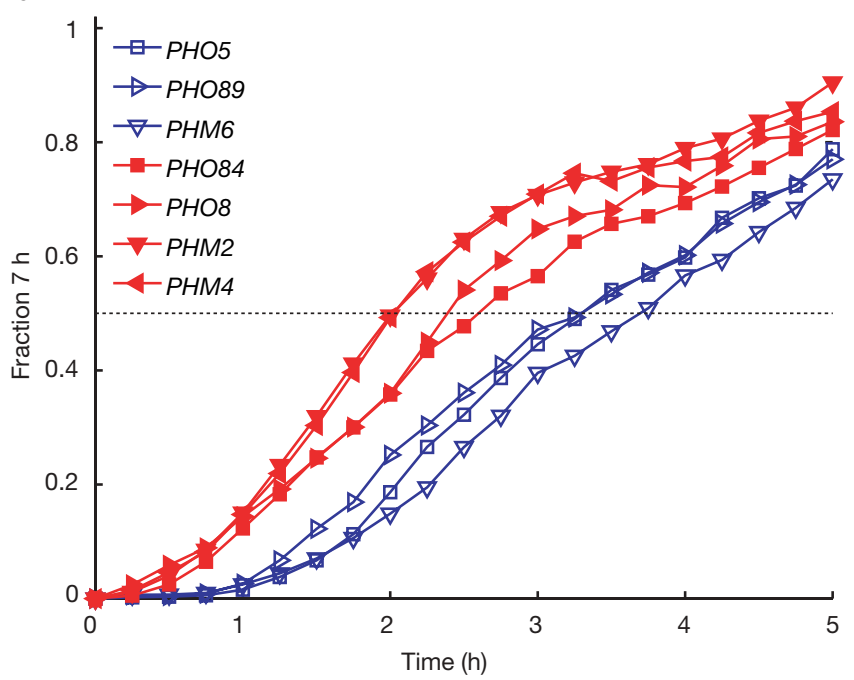

high-affinity motif), light-blue ovals represent low-affinity motifs that are not evolutionarily conserved (Supplementary Fig. 5), and the $x$ axis units reference promoter coordinates with respect to translation start $(\mathrm{ATG}=1)$. In a are $\mathrm{PHO}$ promoters with an accessible low-affinity Pho4 site; in $\mathbf{b}$ are promoters with at least one accessible high-affinity Pho4 site. c, Steady-state transcriptional response of $\mathrm{PHO}$ target genes to $\mathrm{P}_{\mathrm{i}}$. Error bars are interquartile ranges (see Fig. 1b). d, Induction kinetics in $P_{i}$ starvation.

high-affinity sites are substantially induced in intermediate $\mathrm{P}_{\mathrm{i}}$ is physiologically relevant: the products of first-response genes like PHO84 and PHM4 allow the cell to take up environmental $\mathrm{P}_{\mathrm{i}}$ and mobilize internal reserves before a second-order energy commitment is made in starvation conditions to upregulate PHO5 and other $\mathrm{P}_{\mathrm{i}}$ scavenging components ${ }^{14}$. Chromatin renders the signal required for promoter induction sensitive to fewer sites (that is, those exposed), while allowing nucleosomal sites to influence maximum transcriptional output once the promoter becomes open (Fig. 4b). The threshold of activation and dynamic range thus become functionally decoupled, which would not be the case if all binding sites were exposed.

These observations indicate that the interplay between chromatin structure and binding-site affinity may allow different eukaryotic promoters regulated by the same factor to interpret and respond to

Figure 3 | Maximum expression of PHO5 promoter variants. Maximal induction levels of $\mathrm{PHO} 5$ promoter variants measured from strains containing a deletion of the $P H O 80$ gene. The dotted vertical line references the expression output of the wild-type promoter. Data points represent mean \pm s.d. from triplicate measurements; a.u., arbitrary units. 
a



b

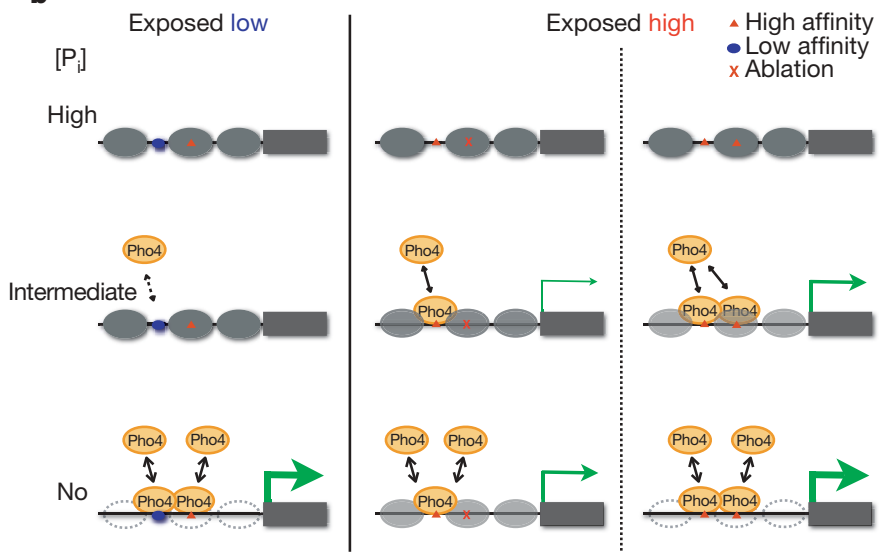

Figure 4 | Pho4 binding in vivo to PHO promoters, and model of thresholddynamic range decoupling. a, Chromatin immunoprecipitation of Pho4 to PHO target genes and the $\mathrm{H} 4$ PHO5 promoter variant (see Fig. 1a) shows differential occupancy in intermediate $\mathrm{P}_{\mathrm{i}}$ conditions at promoters with an exposed high- $(\mathrm{H})$ versus low $(\mathrm{L})$-affinity site. Data points represent mean \pm s.d. from at least three independent experiments. $\mathbf{b}$, Schematic depicting a possible mechanism that decouples promoter induction threshold from dynamic range. In high $P_{i}$ (top row), nucleosomes are fully assembled; in intermediate $P_{i}$ (middle row), substantial Pho4 occupancy occurs only at promoters with exposed high-affinity sites, resulting in chromatin remodelling and transcription commensurate to the total Pho4 recruited; and in $\mathrm{P}_{\mathrm{i}}$ starvation (bottom row), saturating Pho4 activity results in remodelling and maximum expression at all promoters. Nucleosome occupancy is indicated by the opacity of grey ovals, Pho4 by yellow ovals, and the amount of transcription by the thickness of the green arrows. See text for description.

cellular signals uniquely. One implication of this finding is that future models of gene expression will require knowledge both of motifs and nucleosome positions. Additionally, the behaviour of $\mathrm{PHO}$ geneslargely a function of a single transcription factor-will likely prove simple compared with combinatorially controlled genes. But even with the nonlinearities created by multiple transcription factors, it will be fascinating to ascertain if similar architectural principles of promoter chromatin are conserved in other pathways and organisms as a mechanism for quantitatively configuring gene expression.

\section{METHODS SUMMARY}

Promoter variants. Pho4-binding sites or motif ablations were introduced into haploid S. cerevisiae at the chromosomal PHO5 locus. Additionally, the PHO5 coding region was completely substituted with the yeast-enhanced green fluorescent protein $(y e G F P 1)^{30}$ reporter using targeted gene replacement. To assess maximal induction levels, a derivative series of strains was created harbouring both the PHO5 promoter mutations and a deletion of the $\mathrm{PHO} O \mathrm{O}$ gene.

Heterozygous transcriptional reporters. For representative $P H O$ genes (except PHO84, where PHO84pr-yeGFP1 was placed ectopically), one allele in diploid S. cerevisiae was replaced with $y e G F P 1$ at the chromosomal locus.
Growth conditions. For dose-response profiles, strains were grown in 0-50 mM extracellular $P_{i}$ for $18 \mathrm{~h}$ to reach steady-state expression. For induction kinetics, strains grown overnight in $10 \mathrm{mM} \mathrm{P}_{\mathrm{i}}$ were subjected to time-course measurements after transfer into $0 \mathrm{mM} \mathrm{P}_{\mathrm{i}}$. For measurements of maximum induction levels, pho804 strains were grown $18 \mathrm{~h}$ in $10 \mathrm{mM} \mathrm{P}$ to optical density $\left(\mathrm{OD}_{600}\right)$ $0.05-0.1$.

Flow cytometry. Fluorescence distributions were read using a Becton, Dickinson LSR II with $488 \mathrm{~nm}$ laser and calibrated to fluorescent polystyrene microspheres to control for experimental variation. Autofluorescence was assessed in all experiments by inclusion of a strain lacking yeGFP1. Data analysis was done using MATLAB.

Promoter chromatin maps. Mononucleosome-length DNA purified from micrococcal nuclease treated spheroplasts was assayed by quantitative polymerase chain reaction using primer sets that tile approximately 1 kilobase of each promoter region.

Chromatin immunoprecipitation. Cells were grown in high $(10 \mathrm{mM})$, intermediate $(100 \mu \mathrm{M})$ or no $(0 \mu \mathrm{M}) \mathrm{P}_{\mathrm{i}}$ medium for $2.5 \mathrm{~h}$, and fixed in $1 \%$ formaldehyde. Pho4 fold enrichment over POL1 in segments including the nucleosome-free region of each promoter was measured by quantitative polymerase chain reaction, and further re-normalized to the maximal fold enrichment observed in no $\mathrm{P}_{\mathrm{i}}$.

Full Methods and any associated references are available in the online version of the paper at www.nature.com/nature.

Received 24 October 2007; accepted 22 February 2008. Published online 16 April 2008.

1. Kornberg, R. D. \& Lorch, Y. Twenty-five years of the nucleosome, fundamental particle of the eukaryote chromosome. Cell 98, 285-294 (1999).

2. Narlikar, G. J., Fan, H. Y. \& Kingston, R. E. Cooperation between complexes that regulate chromatin structure and transcription. Cell 108, 475-487 (2002).

3. Khorasanizadeh, S. The nucleosome: from genomic organization to genomic regulation. Cell 116, 259-272 (2004).

4. Yuan, G. C. et al. Genome-scale identification of nucleosome positions in $S$. cerevisiae. Science 309, 626-630 (2005).

5. Sekinger, E. A., Mogtaderi, Z. \& Struhl, K. Intrinsic histone-DNA interactions and low nucleosome density are important for preferential accessibility of promoter regions in yeast. Mol. Cell 18, 735-748 (2005).

6. Buck, M. J. \& Lieb, J. D. A chromatin-mediated mechanism for specification of conditional transcription factor targets. Nature Genet. 38, 1446-1451 (2006).

7. Liu, X., Lee, C. K., Granek, J. A., Clarke, N. D. \& Lieb, J. D. Whole-genome comparison of Leu3 binding in vitro and in vivo reveals the importance of nucleosome occupancy in target site selection. Genome Res. 16, 1517-1528 (2006).

8. Workman, J. L. Nucleosome displacement in transcription. Genes Dev. 20, 2009-2017 (2006).

9. Miller, J. A. \& Widom, J. Collaborative competition mechanism for gene activation in vivo. Mol. Cell. Biol. 23, 1623-1632 (2003).

10. Kaffman, A., Herskowitz, I., Tjian, R. \& O'Shea, E. K. Phosphorylation of the transcription factor $\mathrm{PHO} 4$ by a cyclin-CDK complex, $\mathrm{PHO} 80-\mathrm{PHO} 85$. Science 263, 1153-1156 (1994).

11. O'Neill, E. M., Kaffman, A., Jolly, E. R. \& O'Shea, E. K. Regulation of PHO4 nuclear localization by the PHO80-PHO85 cyclin-CDK complex. Science 271, 209-212 (1996).

12. Komeili, A. \& O'Shea, E. K. Roles of phosphorylation sites in regulating activity of the transcription factor Pho4. Science 284, 977-980 (1999).

13. Magbanua, J. P., Fujisawa, K., Ogawa, N. \& Oshima, Y. The homeodomain protein Pho2p binds at an A/T-rich segment flanking the binding site of the basichelix-loop-helix protein Pho4p in the yeast PHO promoters. Yeast 13, 1299-1308 (1997).

14. Springer, M., Wykoff, D. D., Miller, N. \& O'Shea, E. K. Partially phosphorylated Pho4 activates transcription of a subset of phosphate-responsive genes. PLoS Biol. 1, E28 (2003).

15. Ogawa, N. et al. Structure and distribution of specific cis-elements for transcriptional regulation of PHO84 in Saccharomyces cerevisiae. Mol. Gen. Genet. 249, 406-416 (1995).

16. Vogel, K., Horz, W. \& Hinnen, A. The two positively acting regulatory proteins $\mathrm{PHO} 2$ and $\mathrm{PHO} 4$ physically interact with $\mathrm{PHO} 5$ upstream activation regions. Mol. Cell. Biol. 9, 2050-2057 (1989)

17. Ogawa, N., Hayashi, N., Saito, H., Noguchi, K. \& Yamashita, Y. in Phosphate in Microorganisms: Cellular and Molecular Biology (eds Torriani-Gorini, A., Yagil, E. \& Silver, S.) 56-62 (American Society for Microbiology, Washington DC, 1994).

18. Almer, A., Rudolph, H., Hinnen, A. \& Horz, W. Removal of positioned nucleosomes from the yeast $\mathrm{PHO} 5$ promoter upon $\mathrm{PHO} 5$ induction releases additional upstream activating DNA elements. EMBO J. 5, 2689-2696 (1986).

19. Barbaric, S., Munsterkotter, M., Goding, C. \& Horz, W. Cooperative Pho2-Pho4 interactions at the $\mathrm{PHO} 5$ promoter are critical for binding of Pho4 to UASp1 and for efficient transactivation by Pho4 at UASp2. Mol. Cell. Biol. 18, 2629-2639 (1998). 
20. Korber, P., Luckenbach, T., Blaschke, D. \& Horz, W. Evidence for histone eviction in trans upon induction of the yeast $\mathrm{PHO} 5$ promoter. Mol. Cell. Biol. 24, 10965-10974 (2004)

21. Fisher, F. \& Goding, C. R. Single amino acid substitutions alter helix-loop-helix protein specificity for bases flanking the core CANNTG motif. EMBO J. 11, 4103-4109 (1992)

22. Maerkl, S. J. \& Quake, S. R. A systems approach to measuring the binding energy landscapes of transcription factors. Science 315, 233-237 (2007).

23. Svaren, J., Schmitz, J. \& Horz, W. The transactivation domain of Pho4 is required for nucleosome disruption at the PHO5 promoter. EMBO J. 13, 4856-4862 (1994).

24. Venter, U., Svaren, J., Schmitz, J., Schmid, A. \& Horz, W. A nucleosome precludes binding of the transcription factor $\mathrm{Pho} 4$ in vivo to a critical target site in the $\mathrm{PHO} 5$ promoter. EMBO J. 13, 4848-4855 (1994).

25. Thomas, M. R. \& O'Shea, E. K. An intracellular phosphate buffer filters transient fluctuations in extracellular phosphate levels. Proc. Natl Acad. Sci. USA 102, 9565-9570 (2005).

26. Boeger, H., Griesenbeck, J., Strattan, J. S. \& Kornberg, R. D. Nucleosomes unfold completely at a transcriptionally active promoter. Mol. Cell 11, 1587-1598 (2003).

27. Adkins, M. W., Howar, S. R. \& Tyler, J. K. Chromatin disassembly mediated by the histone chaperone Asf1 is essential for transcriptional activation of the yeast PHO5 and PHO8 genes. Mol. Cell 14, 657-666 (2004).

28. Adkins, M. W. \& Tyler, J. K. Transcriptional activators are dispensable for transcription in the absence of Spt6-mediated chromatin reassembly of promoter regions. Mol. Cell 21, 405-416 (2006).
29. Dhasarathy, A. \& Kladde, M. P. Promoter occupancy is a major determinant of chromatin remodeling enzyme requirements. Mol. Cell. Biol. 25, 2698-2707 (2005).

30. Cormack, B. P. et al. Yeast-enhanced green fluorescent protein (yEGFP)a reporter of gene expression in Candida albicans. Microbiology 143, 303-311 (1997)

Supplementary Information is linked to the online version of the paper at www.nature.com/nature.

Acknowledgements We thank: S. J. Maerkl and S. R. Quake for sharing Pho4-binding data; J. S. Weissman, H. Li, R. Losick, B. Stern and present and former members of the O'Shea laboratory for discussion and commentary on the

manuscript; B. S. Margolin for strain EY1995 and assistance with cell sorting; and B. E. Shakhnovich for assistance with motif analysis. Support was provided by the National Institutes of Health, the Howard Hughes Medical Institute, the David and Lucile Packard Foundation (E.K.O.), and the Burroughs Wellcome Fund (F.H.L.).

Author Contributions F.H.L., D.J.S. and E.K.O. designed the experiments. D.J.S made initial promoter variants and preliminary measurements of expression behaviour and nucleosome positions. F.H.L. conducted further strain construction, kinetic and steady-state expression measurements, flow cytometry, nucleosome mapping, chromatin immunoprecipitation and computational analysis. F.H.L. and E.K.O. wrote the manuscript.

Author Information Reprints and permissions information is available at www.nature.com/reprints. Correspondence and requests for materials should be addressed to E.K.O. (erin_oshea@harvard.edu). 


\section{METHODS}

Identification of Pho4 motifs. Analysis by position-specific scoring matrix was used to identify systematically Pho4-binding sites among our selection of chromatin-mapped $P H O$ promoters. From the $256 \Delta \Delta G_{\text {full }}$ values measured by Maerkl and Quake of Pho4 binding to their NNNNGTG DNA oligonucleotide library ${ }^{22}$, the corresponding probabilities of binding were computed to produce an initial position-specific scoring matrix for the four base positions representing the E-box $5^{\prime}$ half-site and flanking base (that is, permuted positions only). To reflect the homodimeric nature of Pho4 binding to DNA ${ }^{31}$ and the optimality of palindromic motifs ${ }^{22}$, these base probabilities were reverse-complemented to fill the $3^{\prime}$ half-site plus flanking position to produce a full octameric positionspecific scoring matrix.

Based on the resulting binding-probability profiles of $\mathrm{PHO}$, $\mathrm{PHO} 4 \mathrm{and}$ PHO8 (Supplementary Fig. 4a, d, e, respectively), the Pho4-promoter interactions of which have been characterized by in vitro DNase I protection assays $^{15,17,24,32}$, a noise threshold for likely Pho4 regulatory elements was determined by selecting the weakest probability matching an experimentally verified footprint. To distinguish high- from low-affinity motifs, a second threshold was established by comparing the binding profiles of $\mathrm{PHO} 5$ and $\mathrm{PHO} 4$ with in vitro competition footprinting experiments from the literature ${ }^{15,17}$, and selecting the most stringent probability necessary to recapitulate validated high-affinity sites. Finally, putative Pho4 sites were plotted on multiple alignments of Saccharomyces orthologues to assess evolutionary conservation (Supplementary Fig. 5) on the assumption that conserved sites indicate a higher likelihood of function ${ }^{33,34}$.

Flow cytometry analysis. To control for day-to-day instrument variation, a fivepoint calibration sample of fluorescent polystyrene microspheres was read at the start and end of each experiment. A weighted least-squares fit to the calibration points established a standard curve spanning an approximate 1,000-fold dynamic range that allowed for accurate quantitative comparison across different experiments. Signal area data from the fluorescein isothiocyanate channel (FITC-A) for 10,000 cells were collected from all samples and interpolated off these standard curves before further analysis. Any reference to 'calibrated fluorescence' in this study refers to these standardized units.

Although samples were sonicated before flow cytometry to reduce cell aggregation, simple rectangular gating in the forward-scatter and side-scatter channels was performed to further reduce variability. An automated approach was implemented whereby data points outside the 0.1 and 0.85 quantiles in either forward scatter and side scatter were eliminated. This worked well empirically to eliminate debris and any remaining cell aggregates.

Although autofluorescence was minimized in all synthetic media experiments by the addition of supplementary adenine and tryptophan, a wild-type strain lacking yeGFP1 was included in all experiments to assess the level of fluorescence specific to gene expression. Instead of doing subtraction with fitted distribution parameters estimated from the yeGFP $1^{-}$and transcriptional reporter samples, a randomly permuted vector subtraction of autofluorescence from the transcriptional reporter distributions was performed instead. This numerical approach was deemed more unbiased as assumptions of normality or distribution symmetry would not have to be made, and may have been particularly suited in situations where population behaviour was highly skewed (for example, during induction in $\mathrm{P}_{\mathrm{i}}$ starvation conditions).

Two approaches were taken to estimate times of half-maximal induction (Supplementary Tables 1 and 2). The first was a simple linear interpolation of the time needed to reach a level halfway between the median expression observed at 0 and $7 \mathrm{~h}$ (Figs $1 \mathrm{c}$ and $2 \mathrm{~d}$ ). However, as interpolated half-times are highly sensitive to maximal induction levels, a second approach was taken for comparison where the unscaled kinetic data were subjected to a nonlinear least squares fit of a Hill equation with three freely varying parameters: $n$ (Hill coefficient), $Y_{\max }$ (asymptotic maximal value) and $T_{\text {half }}$ (half maximal time). Because $Y_{\max }$ is a freely varying parameter, $T_{\text {half }}$ is not constrained to an arbitrary maximum and is free to adopt the value that best approximates the continuous underlying behaviour. In general, the fits gave normally distributed residuals centred around zero and low root mean squared errors (fit standard errors), indicating that the Hill model described the kinetic data well.

31. Shimizu, T. et al. Crystal structure of $\mathrm{PHO} 4 \mathrm{bHLH}$ domain-DNA complex: flanking base recognition. EMBO J. 16, 4689-4697 (1997).

32. Barbaric, S., Fascher, K. D. \& Horz, W. Activation of the weakly regulated PHO8 promoter in S. cerevisiae: chromatin transition and binding sites for the positive regulatory protein PHO4. Nucleic Acids Res. 20, 1031-1038 (1992).

33. Kellis, M., Patterson, N., Endrizzi, M., Birren, B. \& Lander, E. S. Sequencing and comparison of yeast species to identify genes and regulatory elements. Nature 423, 241-254 (2003).

34. Kent, W. J. et al. The human genome browser at UCSC. Genome Res. 12, 996-1006 (2002). 